\title{
Chromocult Coliform agar and duplex PCR assays as methodologies for tracking Escherichia coli K12 in industrial biotechnological processes
}

\author{
Gisele d'Angelis Antunes', Janine Aparecida Correia Durães Gandra², Elane Alquimim Moreira', \\ Wesley Cézar Silva Machado', Shirley da Silva Gomes Magalhães', Mauro Aparecido de Sousa Xavier², \\ Alessandra Rejane Ericsson de Oliveira Xavier ${ }^{2 *}$ \\ 'Novo Nordisk Produção Farmacêutica do Brasil, ALP Production, Montes Claros, MG, Brazil. \\ ${ }^{2}$ State University of Montes Claros, Center for Biological and Health Sciences, Graduate Program in Biotechnology, Microbiology Laboratory, Montes \\ Claros, MG, Brazil.
}

\section{ARTICLE INFO \\ Article history: \\ Received on: 19/12/2017 \\ Accepted on: 06/02/2018 \\ Available online: 30/03/2018}

\author{
Key words: \\ Chromocult ${ }^{\circledR}$ Coliform \\ Agar, Filter Membrane, \\ Escherichia coli $\mathrm{K} 12$, \\ duplex PCR, Genetically \\ Modified Organism, \\ Detection.
}

\begin{abstract}
The objective of this work was to establish support for the validation of the microbiological method associated with the duplex PCR assay for Escherichia coli K12 in waste and intermediate products of an industrial biotechnological process. For this purpose, samples of Escherichia coli K12 from critical control points known as Killing System (KS) and Germ Filtration (GF) were analyzed. Experiments to determine the microbial load of E. coli K12 based on the direct plating and filter membrane methods revealed the absence of this microorganism in the KS and GF samples. The chromogenic medium was able to recover strains of $E$. coli inoculated intentionally in GF samples, but the same result was not possible in samples from KS. The robustness of the incubation time of the KS and GF samples were calculated by the ANOVA test and the results showed no statistically significant difference between them ( $p$-value 0.181 and 0.733). Duplex PCR analysis was shown to be able to differentiate a standard strain of Escherichia coli ATCC 8739 from Escherichia coli K12. The results will be used as a guideline for validation of a method for $E$. coli K12 tracking in an industrial biotechnological process.
\end{abstract}

\section{INTRODUCTION}

The technology of manufacturing biopharmaceuticals and other biomolecules can be divided into upstream and downstream processes. Upstream processes range from the selection of production strains, culture media, growth parameters to the optimization of ideal conditions for biomolecule production. Downstream phases include all steps required for the purification of a biological product

\footnotetext{
* Corresponding Author Alessandra Rejane Ericsson de Oliveira Xavier, State University of Montes Claros, Center for Biological and Health Sciences, Graduate Program in Biotechnology, Microbiology Laboratory, Montes Claros, MG, Brazil.E-mail: ericsson_aerc@yahoo.com.br
}

from the culture medium to the final purified product. It involves multiple steps to obtain the target molecule and remove from the host cell impurities (host cell proteins, DNA, among others), process-related impurities (buffers, defoamers, leaching binders and others) and products (aggregates and fragments, among others). Each purification step must be able to remove one or more classes of impurities (Azevedo et al., 2009; Rathore and Kapoor, 2015; Jozala et al., 2016). Downstream processes include (1) initial recovery (extraction or isolation), (2) purification (removal of major contaminants), and (3) cleaning (removal of specified contaminants and undesired forms of the target biomolecule that may have been formed during isolation and purification) (Rosa et al., 2010; Fields et al., 2016; Jozala et al., 2016).

The production of recombinant proteins in biotechnological industries can be carried out with genetically 
modified organisms (GMOs), including those derived from Escherichia coli K12 (Simões et al., 2016; Gandra et al., 2017). Different lineages of Escherichia coli K12 are used in research and development in the field of biotechnology, as well as in industrial production (Kuhnert et al., 1995; Simões et al., 2016; Azpiroz and Lavinã, 2017). In Brazil, the national biosafety policy with regard to genetically modified organisms (GMOs, which includes Escherichia coli K12) is regulated by the National Technical Commission on Biosafety (Conceição et al., 2006; Simões et al., 2016).

According to Brazilian legislation, the risks and effects of upstream and downstream biotechnological processes arising from the release of GMOs into the environment must be evaluated. In this sense, research institutions and industries active in the biotechnology field must develop methods for the detection of GMOs in developed processes, in order to identify them in case of accidental release to the environment (Miralles et al., 2009; Costa et al., 2011; Simões et al., 2016).

The detection of Escherichia coli can be affected through microbiological methods, including those based on culture media using chromogenic substrates (Turner et al., 2000; Finney et al., 2003; Maheux et al., 2014). Chromocult ${ }^{\circledR}$ Chromogenic Agar (CCA) is a simple medium containing two chromogenic substrates for the detection of total coliforms and Escherichia coli. The chromogenic substrates are X-GLUC (5-bromo-4-chloro-3-indoxyl- $\beta$-D-glucoronide), 96\% specific for identification of Escherichia coli (Frampton and Restaino, 1993; Turner et al., 2000) and Salmon-GAL (6-chloro-3-indoxyl- $\beta$-Dgalactoside). Because Escherichia coli present a positive reaction to both substrates, their colonies in the CCA medium vary from dark blue to violet. Members of the family Enterobacteriaceae which do not encode $\beta$-Glucuronidase (Citrobacter, Enterobacter and Klebsiella), being positive for $\beta$-galactosidase, present colonies varying from salmon to red in this culture medium. Others which are $\beta$-Glucuronidase positive, but $\beta$-galactosidase negative, such as Shigella, Salmonella and Yersinia produce turquoise blue colonies. Gram-negative bacteria that do not encode the $\beta$-Glucuronidase and $\beta$-galactosidase enzymes produce clear colonies in the CCA medium (Turner et al., 2000; Finney et al., 2003). Gram-positive bacteria and other non-enteric bacteria are inhibited by the presence of Tergitol-7. Further confirmation of the Escherichia coli can be accomplished by detecting indole by adding tryptophan to the medium, thereby increasing the reliability of Escherichia coli detection (Finney et al., 2003).

Alternatively, the identification of Escherichia coli and other enteric bacteria can be obtained by molecular methods such as PCR (Polymerase Chain Reaction) for the detection of the pal gene which encodes a peptidoglycan-associated lipoprotein among the enterobacteria (Kuhnert et al., 1995). Genetic identification of strains derived from Escherichia coli K12 can be obtained in a differentiated manner based on PCR detection of the presence of an IS5 insert in the gene cluster encoding Rhamnose transferase. The molecular method used presents a useful tool in the identification of Escherichia coli K12 for monitoring strains that are used as biologically safe vehicles in biotechnology research, development and production processes (Kuhnert et al., 1995; Simões et al., 2016). Simões et al. (2016) developed a method based on PCR for detection of Escherichia coli K12 carrying an ampicillin resistance gene in a pond of an effluent treatment station. The molecular method developed was preceded by a microbiological method, scattering in plates depending on cultivation, since the analysis is performed in order to monitor the presence of viable microorganisms transporting a gene marker for $E$. coli $\mathrm{K} 12$ according to recommendations of the National Biosafety technical Commission (Simões et al., 2016).

The biotechnology industry where this work was conducted produces a recombinant protein on a large scale using Escherichia coli $\mathrm{K} 12$ as host. Any contaminated liquid or solid waste is decontaminated before being discarded, along with any material or equipment that has come into contact with the GMO, according to current legislation (BRASIL, 2006; Gandra et al., 2017).

Microbiological methodologies associated with the molecular that allow the monitoring of the presence of the host Escherichia coli $\mathrm{K} 12$ in residues generated by industrial biotechnological processes are mandatory in Brazil. The objective of this study was to establish bases for validation of a microbiological methodology using the selective medium CCA associated with the duplex PCR assay for tracking the host Escherichia coli K12 in waste and intermediate product of an industrial biotechnological process.

\section{MATERIALS AND METHODS}

\section{Bacterial lines and culture media}

The strains used are Escherichia coli ATCC 8739 and Escherichia coli W3110 carrying genotyped plasmid pUC18, according to Simões et al. (2016). Pure cultures of the microorganisms used were previously quantified (approximately 5.0 of $10^{9} \mathrm{CFU} / \mathrm{ml}$ ), preserved in $20 \%$ glycerol and stored at $-80^{\circ} \mathrm{C}$. The media Chromocult巴 Coliform Agar (Chromocult; Merck, UK) and Trypticase Soy Agar (TSA, BD-Difco ${ }^{\mathrm{TM}}$, USA) were prepared according to manufacturers' instructions. For selection of Escherichia coli W3110, with the presence of plasmid pUC18 the Chromocult ${ }^{\circledR}$ Coliform Agar medium was supplemented with ampicillin (Sigma-Aldrich, $200 \mu \mathrm{g} / \mathrm{ml}$ ).

\section{Description and characterization of sample collection points}

The samples analyzed in this study were composed of residual liquids and intermediate product obtained from a biotechnological process. The location and description of sample collection points are shown in Figure 1. Points 1 and 2 indicated in this figure correspond respectively to Killing System (physical system inactivator) and Sterilizing Filtration (stage for removal of the residual GMO from the final product). Samples were collected in triplicate from the Killing System (point 1) and Sterilizing Filtration (point 2) during the production of three different batches of a recombinant protein produced on an industrial scale.

\section{Determination of the microbial load present in residuals and the intermediate product of an industrial biotechnological process}

The amount of $100 \mu \mathrm{L}$ samples collected from point 1 (Figure 1) were inoculated in triplicate, using an automatic pipette directly on plates with the presence of CCA medium supplemented with ampicillin. The inoculation of $100 \mu \mathrm{L}$ of saline 
solution used directly on plates containing the same medium was used as negative control. As a positive control, a suspension was used with a concentration of $100 \mathrm{CFU} / 100 \mu \mathrm{l}$ of Escherichia coli $\mathrm{W} 3110$ (Figure 2A). Plates were incubated at $32.5 \pm 2.5^{\circ} \mathrm{C}$ for 24 hours. The whole procedure was carried out in a biological safety cabinet (Telstar, Bio II Advance) with due precautions against microorganisms from the air.

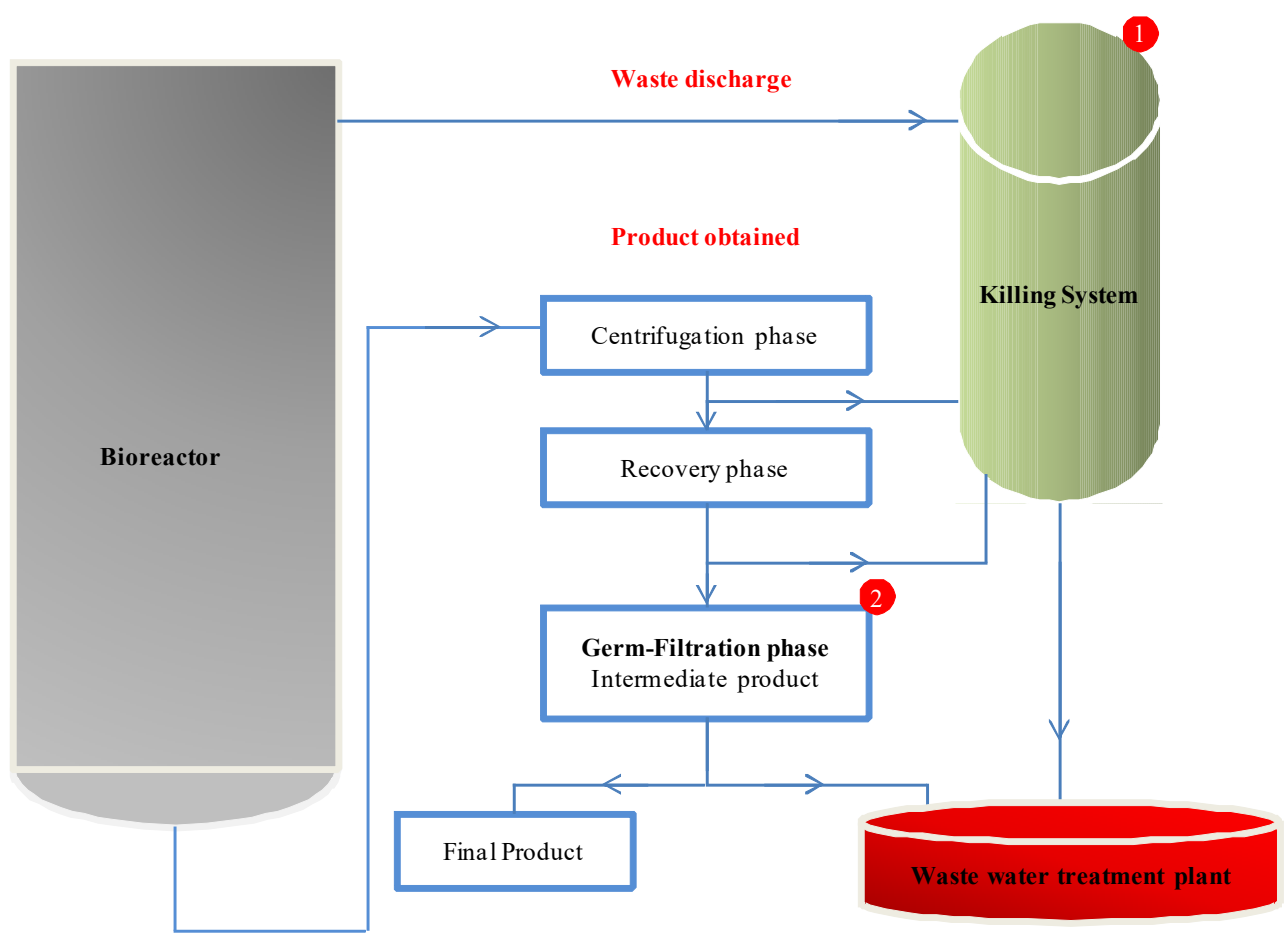

Fig. 1: Disposal flow and treatment of the wastes generated during and after the production of final recombinant product on an industrial scale. Point 1 corresponds to the Killing System, a system of thermal inactivation of residual liquids with a potential risk of the presence of genetically modified organisms. It is composed of tanks, which receive residual liquids from the industrial biotechnological process. Point 2 corresponds to the sterilizing filtration step (Germ Filtration) for removal of any microbial residue from the final product.
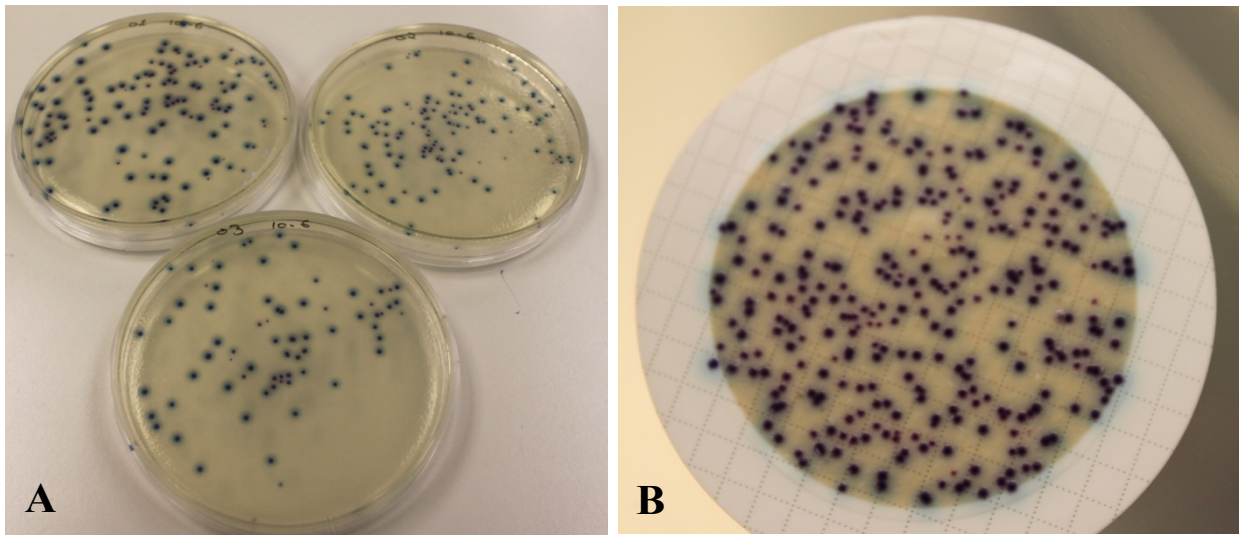

Fig. 2: Aspect of the growth of the colonies of Escherichia coli K12 in the medium Chromocult Cromogenic Agar (CCA). Panel A: Direct plating (scattering method) of the bacterial inoculum containing $100 \mathrm{CFU} / 100 \mu \mathrm{l}$. Panel B: Filtration of the bacterial inoculum containing between $100 \mathrm{CFU} / 100 \mathrm{ml}$ (filter membrane method).

In triplicate, $100 \mathrm{~mL}$ samples collected from point 2 (Figure 1) with the aid of a vacuum filtration system (EZ-STREAM vacuum pump and manifold EZ-FIT TRES, Merck Millipore) coupled to a membrane with $0.45 \mu \mathrm{m}$ pores. After filtration the membranes were added directly onto plates with the presence of CCA medium supplemented with ampicillin and incubated at $32.5 \pm 2.5^{\circ} \mathrm{C}$ for 24 hours. The same methodology was followed for the negative control, where $100 \mathrm{~mL}$ of the saline solution was filtered. As a positive control, $100 \mathrm{~mL}$ of saline was intentionally contaminated with a concentration of $100 \mathrm{CFU} / 100 \mathrm{ml}$ of Escherichia coli W3110 (Figure 2B).

To determine the robustness of the incubation time the CFUs present in the plates with samples from points 1 and 2 were counted with the aid of the colony counter (CP600 plus, Phoenix) at times of 18, 21 and 24 hours. The results of the CFU count on the plates were recorded in an Excel spreadsheet and analyzed statistically. 


\section{Contamination and recuperation of Escherichia coli W3110} in samples of the Killing System and Germ Filtration

A standardized concentration of $100 \mathrm{CFU} / 100 \mathrm{ml}$ of Escherichia coli $\mathrm{W} 3110$ was used in a contamination and recovery experiment on samples collected and previously sterilized from points 1 and 2 as indicated in Figure 1. Samples from point 1 after being intentionally contaminated with Escherichia coli W3110 were inoculated according to the direct plating method mentioned in the previous session. The reason for choosing the method used for the Killing System was because they have high viscosity, which makes filtration unfeasible. Samples from point 2 were subjected to the membrane filtration method according to the United States Pharmacopoeia (USP, 2016). Positive and negative controls were performed as mentioned in the previous session. To determine the robustness of the incubation time the CFUs present in the plates with samples from points 1 and 2 were counted with the aid of the colony counter (CP600 plus, Phoenix) at times of 18, 21 and 24 hours. The results of counting CFUs on the plates were recorded in Excel spreadsheet programmed to automatically calculate the recovery rate.

\section{Calculation of Recovery rate and working range}

The recovery rate $(\% \mathrm{R})$ of the microorganism intentionally inoculated in CCA culture medium was calculated by the following equation:

$\% \mathrm{R}=($ Mean of the test plate $) /($ Mean of control plate $) \times 100$

The criterion was that the mean count of the microorganism should be in the range of 50 (lower limit) to $200 \%$ (upper limit) according to internal laboratory procedures and in accordance with the United States Pharmacopoeia (USP, 2016).

\section{Duplex PCR assay}

Colonies grown in TSA and CCA media supplemented with ampicillin were transferred directly to microtubes containing $100 \mu \mathrm{l}$ of sterile saline $(0.9 \% \mathrm{NaCl})$ and centrifuged at $8000 \mathrm{~g}$ for $5 \mathrm{~min}$. The supernatant was discarded and the sediment was resuspended in $100 \mu \mathrm{l}$ of sterile saline $(0.9 \% \mathrm{NaCl})$. The suspension with microbial cells and nucleic acids was diluted 1:10 and $4 \mu \mathrm{l}$ was used as template DNA for duplex PCR. The two distinct strains of Escherichia coli were submitted to the duplex PCR reaction with primers K12-L (TTCCCACGGACATGAAGACTACA), K12-R (ATCCTGCGCACCAATCAACAA), ECPAL-L (GGCAATTGCGGCATGTTCTTCC) and ECPAL-R (CCGCGTGACCTTCTACGGTGAC). The primers K12-L and K12-R generate fragments respectively of 1690 base pairs for E. coli K12 according to Kuhnert et al. (1995). A 280 base pairs fragment is expected with the amplification of ECPAL-L and ECPAL-R primers in any enterobacteriaceae (Kuhnert et al., 1995). All primers were synthesized by Integrated DNA Technology, USA. Reactions were performed in duplex PCR with the $2 \mathrm{x}$ reagent Gotaq Green Master Mix ${ }^{\circledR}$ (Promega, Corporation, USA), $\mathrm{MgCl}_{2}(2.5 \mathrm{mM}), 10 \mu \mathrm{M}$ of each primer and $4 \mu \mathrm{l}$ of microbial extract in a final reaction volume of $50 \mu \mathrm{l}$. The amplification conditions were those indicated by Kuhnert et al. (1995) for each primer. The amplicons were visualized on $1.5 \%$ agarose gel stained with ethidium bromide and photodocumented. As a positive control of the PCR reaction, standard strains were use of E. coli K12 W3110 and E. coli ATCC 8739, maintained in the laboratory. Water was used as a negative control.

\section{Statistical analysis}

The means and standard deviations of the results of the CFU counts and recovery rate obtained in the experiments were calculated. For the analysis of robustness of the incubation time of the samples, the ANOVA statistical test with $95 \%$ significance was performed using Minitab.V16 software. Tables and charts were generated in Excel.

\section{RESULTS AND DISCUSSION}

A total of 36 samples, 18 of which were collected from the Killing System and 18 from the Germ Filtration (points 1 and 2 of Figure 1) were used as a source of microorganism isolation. In addition, CCA supplemented with ampicillin was used in experiments of intentional contamination with E. coli K12 and verification of recovery rate in medium.

Colony growth was not obtained in the samples isolated in points 1 and 2 shown in Figure 1. The results from the 18, 21 and 24-hour plate readings were $0 \mathrm{CFU}$. The positive and negative controls of the test presented results within that expected (growth and absence of growth, respectively). This result was expected and confirms the efficiency of the treatment of industrial liquid waste by the Killing System before being introduced into the Effluent Treatment Station.

Information on nutritional conditions, physical parameters for growth and thermal inactivation of bacteria, among which are those derived from Escherichia coli K12 are well established in the literature (Cornet et al., 2010; Derlinden et al., 2007; Fogolari et al., 2012). According to internal procedures of the biotechnology industry where this study was carried out, the Killing System operates at a temperature of $121^{\circ} \mathrm{C}$ and among the residues received are those containing sodium hydroxide (from tank cleaning), which renders the environment alkaline and makes microbial growth difficult, and especially for E. coli K12.

Samples collected from Germ Filtration were those that underwent the sterilizing filtration process on a $0.22 \mu \mathrm{m}$ filter capable of retaining bacteria which includes $E$. coli K12. The absence of growth of microorganisms in samples from the Germ Filtration also confirms the efficiency of the system in eliminating microorganisms from the final biotech product.

Lineages of $E$. coli are considered standard for measuring the quality of treated waste water from industrial waste (Bower et al., 2005; Frigon et al., 2013). The literature presents references on the microbial load found in waste sent to effluent treatment stations (Wang et al., 2014; Li et al., 2010; Martinez, 2009). Information about validations of industrial systems that use wet steam as a sterilization process are found in the literature (ISO11138-1, 2017; Mannermaa and Yliruusi, 1993) and the microorganism Geobacillus stearothermophilus is the main bioindicator of the efficiency of the sterilization process (HuescaEspitia, 2016; Lundahl, 2003; McLeod, 2017). However, up to the present moment it has not been possible to verify information related to the determination of the surviving microbiota present in industrial scale systems for physical inactivation similar to the one used in this study. The literature revealed that this is the first work that uses a technique based on culture in selective medium 
and genetic analysis by PCR for tracing Escherichia coli K12 in industrial waste before being sent to an effluent treatment plant. In previous work, a methodology for tracking E. coli K12 was standardized; however, the samples came from an effluent treatment plant pond, following upstream and downstream steps of a biotechnological process (Simões et al., 2016).

In order to verify that the Killing System would be conducive to the growth of $E$. coli $\mathrm{K} 12$, samples taken from it were intentionally contaminated with a known concentration of this microorganism (100 CFU). Prior to contamination the samples were autoclaved and inoculated only after cooling to room temperature. Negative and positive controls were used. There was no E. coli $\mathrm{K} 12$ in the contaminated samples seeded in CCA medium and consequently the recovery rate was $0 \%$ compared to the positive control (Figure 3A). In alignment with the result obtained with the experiment for determination of the microbial load of the Killing System it was observed that the environment became inappropriate to the growth of the inoculated microorganism. PH measurements of the three sample batches of the Killing System confirmed an alkaline environment $(\mathrm{pH} \geq 9)$, in addition to the presence of detergent compounds potentially present in the waste disposal and treatment environment.

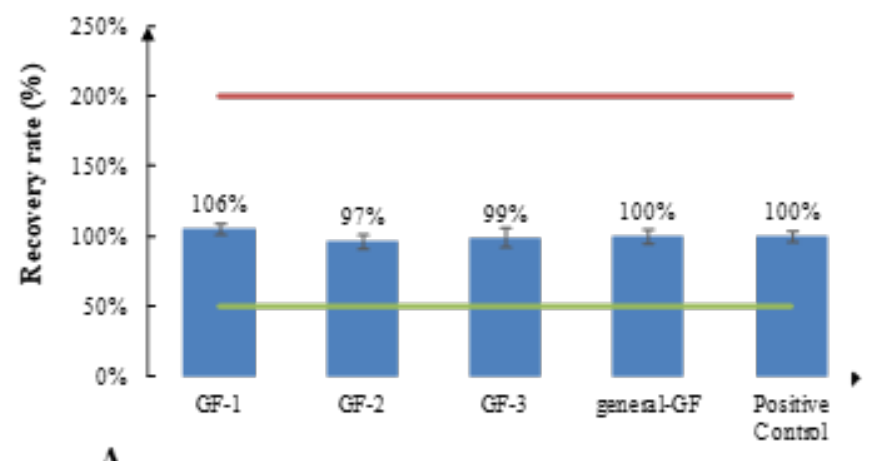

A

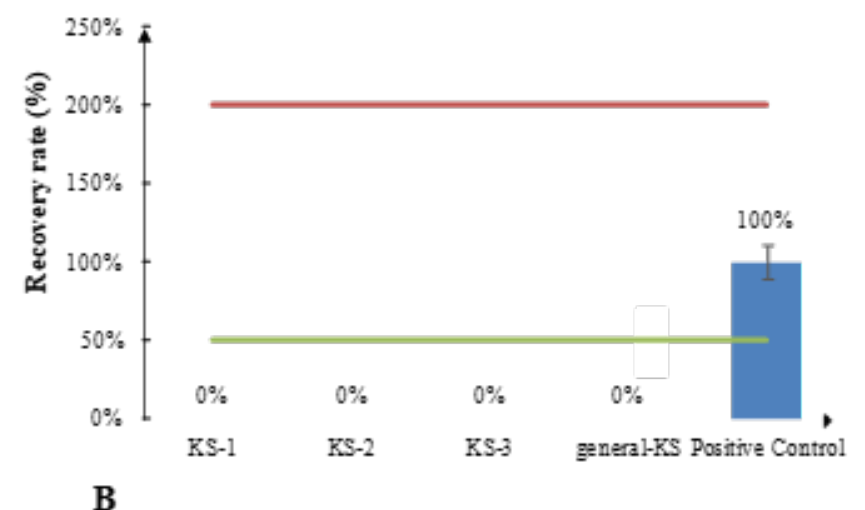

Fig. 3: Percentage of recovery rate of Escherichia coli K12 inoculated into samples from Killing System (KS) and Germ Filtration (GF). Panel A: Samples from 3 different batchess of the contamination/recovery experiment (KS-1 to $\mathrm{KS}-3$ ). The result of the overall mean between the batches is shown in the figure (general-KS), as well as the positive control (E. coli K12 inoculated in saline solution). Panel B: Samples from 3 different batchess of the contamination/ recovery experiment (GF-1 to GF-3). The result of the overall mean between batches is shown in the figure (general-GF) as well as the positive control $(E$. coli $\mathrm{K} 12$ inoculated in saline solution).
Samples of the process of Germ Filtration were also contaminated with $E$. coli $\mathrm{K} 12$ and the results showed that it was possible to recover the amount of this microorganism inoculated within the expected range (Figure 3B). When comparing the test results with the positive control the CCA medium supplemented with ampicillin was able to recover E. coli K12 previously inoculated into samples from Germ Filtration. The results showed that the intermediate product has an environment favorable to microbial growth and especially the E. coli K12. However, the results of the microbial load experiments revealed that the sterilizing filtration step was efficient in eliminating microorganisms during the routine of the biotechnological process.

The robustness of the incubation times of the Killing System and Germ Filtration used in the contamination and recovery tests were statistically evaluated. Although there were slight variations between the 18, 21 and 24-hour counts, there were no statistically significant differences between the above samples. The $p$-value for samples of the Killing System was 0.181 and for Germ Filtration 0.733. Similar results were found in contamination and recovery of $E$. coli $\mathrm{K} 12$ when used in saline solution (Gandra et al., 2017).

Qualitative and quantitative methods for screening for GMOs based on the detection of nucleic acids are described in the literature (Kuhnert et al., 1995; Simões et al., 2016; Cardoso et al., 2017). Although the observed results show the efficiency of the Killing System and Germ Filtration to eliminate or prevent the growth of E. coli K12 in the tested samples, the development of methods for identifying GMOs is a mandatory activity in biotechnological industries (Simões et al., 2016). The need to develop methods capable of differentiating wild $E$. coli (commonly found in industrial waste) from those derived from E. coli $\mathrm{K} 12$ is salutary in biotechnology industries. A duplex PCR assay was therefore standardized in samples of $E$. coli $\mathrm{K} 12$ grown in CCA medium, since it is the culture medium that will be used for screening and tracking of E. coli in discard samples and the final product of the biotechnological process. Figure 4 shows the genetic identification of the strain $E$. coli $\mathrm{K} 12$ where the expected profile for this microorganism was found (Kuhnert et al., 1995; Simões et al., 2016). The lineages E. coli K12 W3110 and E. coli ATCC 8739 presented distinct profiles that could be differentiated from each other by the duplex PCR technique standardized in this study. The 1690 base pair PCR product related to the insertion of the IS5 sequence into the gene encoding for Rhamnose Transferase in Escherichia coli K12 (Kuhnert et al., 1995) was only visualized in the strains of E. coli K12 W3110 (Figure 4). In both strains a fragment of 280 base pairs corresponding to the gene pal, expected marker of the family to which these bacteria belong (Kuhnert et al., 1995).

\section{CONCLUSION}

The method of analysis based on microbiological and molecular techniques for tracking E. coli $\mathrm{K} 12$ in the biotechnological process was developed in this study. The results confirmed the efficiency of the Killing System and Germ Filtration in ensuring that residuals and final product derived from recombinant DNA technology are free of GMOs. The microbiological method used based on direct plating or filter membrane in CCA culture medium was able to promote the 
growth of the strain E. coli K12. The duplex PCR methodology allowed the distinction between a species of E. coli $\mathrm{K} 12$ and one of E. coli ATCC. To our knowledge, this is the first time that a microbiological methodology has been described in the literature using the selective medium CCA associated with the duplex PCR assay for monitoring the presence of the host
Escherichia coli $\mathrm{K} 12$ in the intermediate product and wastes of an industrial biotechnological process. The observed results will serve as a guideline for the validation of a $E$. coli $\mathrm{K} 12$ in routine biotechnological process, as well as in case of accidental disposal of this GMO.

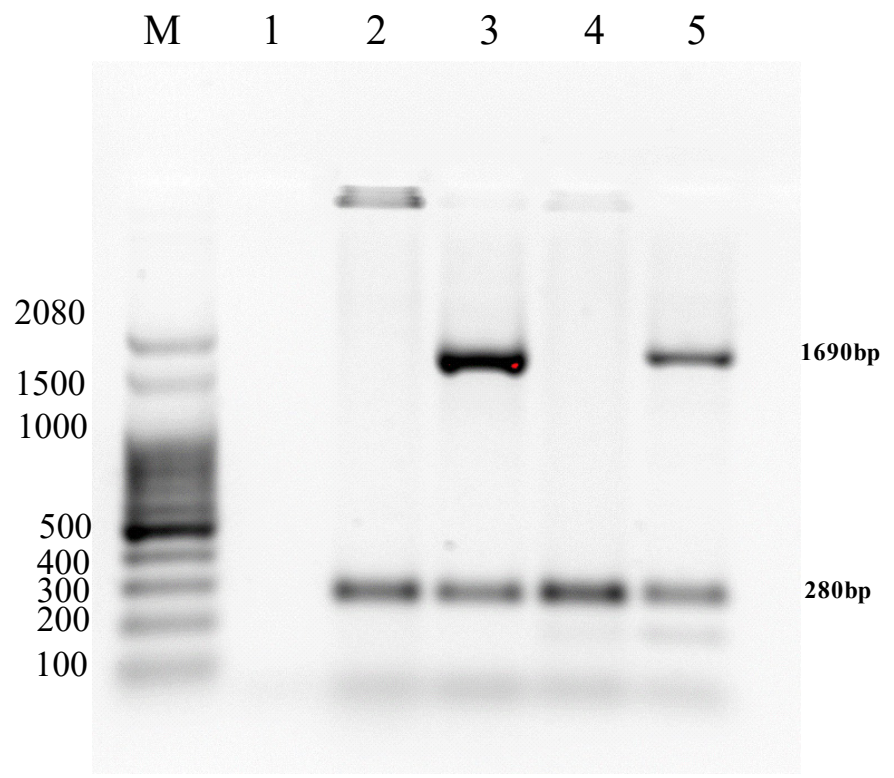

Fig. 4. PCR duplex for identification of E. coli K12 W3110 maintained in the laboratory and subcultured on CCA medium. M: 100 bp molecular weight marker (Ludwig). Line 1: negative control (primers K12L/K12R; ECPAL-L/ECPAL-R, no DNA). Lines 2 and 3: positive controls (Escherichia coli ATCC8739 and Escherichia coli W3110 respectively maintained in TSA medium amplified with primers K12L/K12R; ECPAL-L/ECPAL-R). Lines 4 and 5: Escherichia coli ATCC8739 and Escherichia coli W3110 maintained in CCA medium amplified with primers K12L/K12R; ECPAL-L/ECPAL-R. The sizes of the expected fragments amplified with these primers are shown to the right of the $1.5 \%$ electrophoresis gel.

\section{AUTHORS CONTRIBUTIONS}

Gisele d'Angelis Antunes, Janine Aparecida Correia Durães Gandra, and Alessandra Rejane Ericsson de Oliveira Xavier contributed equally to this work.

\section{ACKNOWLEDGMENTS}

Thanks to the biotechnology company Novo Nordisk Produção Farmacêutica do Brasil, for financial support and to the master's program in Biotechnology at the State University of Montes Claros.

\section{CONFLICT OF INTEREST}

There are no conflicts of interest to declare.

\section{REFERENCES}

Azevedo AM, Rosa PAJ, Ferreira IF, Aires-Barros MR Chromatography-free recovery of biopharmaceuticals through aqueous two-phase processing. Trends Biotechnol, 2009; 27:240-247.

Azpiroz MF, Laviña M. Analysis of RecA-independent recombination events between short direct repeats related to a genomic island and to a plasmid in Escherichia coli K12. PeerJ, 2017; 5: e3293.

Bower PA, Scopel CO, Jensen ET, Depas MM, McLellan SL. Detection of genetic markers of fecal bacteria in Lake Michigan and determination of their relationship to Escherichia coli densities using standard microbiological methods. Appl Environ Microbiol, 2005; 71: 8305-8313.
BRAZIL. Ministry of Science, Technology and Innovation. Technical Biosafety Commission Normative Resolution No. 2 of November 27, 2006. 27/nov 2006. Available at: http://ctnbio.mcti.gov.br. Accessed on: October 31, 2017.

Cardoso L, Cangussu CHC, Nogueira HS, Santos J, Xavier MAS, Oliveira AME, Albuquerque CJB, Xavier AREO. Métodos baseados na detecção de DNA para rastreamento de modificações genéticas em cultivares transgênicos de milho e soja. Caderno de Ciências Agrárias, 2017; volume 9, in press.

Conceição FR, Moreira NA, Binffeld PC. Detecção e quantificação de organismos geneticamente modificados em alimentos e ingredientes alimentares. Cienc Rural, 2006; 36: 315-324.

Cornet I, Van Derlinden E, Cappuyns AM, Van Impe JF. Heat stress adaptation of Escherichia coli under dynamic conditions: effect of inoculum size. Lett Appl Microbiol, 2010; 51: 450-455.

Costa TE, Dias AP, Scheidegger EM, Marin VA. Avaliação de risco dos organismos geneticamente modificados. Cien Saude Colet, 2011; 16: 327-336.

Derlinden EV, Bernaerts K, Van Impe JF. Dynamics of Escherichia coli at elevated temperatures: effect of temperature history and medium. J Appl Microbiol, 2007; 104:438-453.

Fields C, Li P, O’Mahony JJ, Lee GU. Advances in affinity ligand-functionalized nanomaterials for biomagnetic separation. Biotechnol Bioeng, 2016; 113: 11-25.

Finney M, Smullen J, Foster HA, Brokx S, Storey DM. Evaluation of Chromocult coliform agar for the detection and enumeration of enterobacteriaceae from faecal samples from healthy subjects. J Microbiol Methods, 2003; 54: 353-358. 
Fogolari O, Reis CZ, Philippi LS. Determinação de parâmetros cinéticos da inativação térmica de Escherichia coli em lodo de esgoto. Eng Sanit Ambient, 2012; 17: 255-262.

Frampton EW, Restaino L. Methods for Escherichia coli identification in food, water, and clinical samples based on betaglucuronidase detection. J Appl Bacteriol, 1993; 74: 223-233.

Frigon D, Biswal BK, Mazza A, Masson L, Gehra R. Biological and physicochemical wastewater treatment processes reduce the prevalence of virulent Escherichia coli. Appl Environ Microbiol, 2013; 79: 835-844.

Gandra JACD, Machado WCS, Oliveira AME, Nogueira HS, Magalhães SSG, Sanches G, Xavier MAS, Xavier AREO. Comparative analysis between the microbiological filtering and scattering methods in the recovery of Escherichia coli K12 in purposely contaminated saline solution. Revista Unimontes Científica, 2017; 19: 13-24.

Huesca-Espitia LC, Suvira M, Rosenbeck K, Korza G, Setlow B, Li W, Wang S, Li YQ, Setlow P. Effects of steam autoclave treatment on Geobacillus stearothermophilus spores. J Appl Microbiol, 2016; 121: 1300-1311.

International Organization for Standardization: ISO11138-1: Sterilization of health care products - Biological indicators. Part1: General requirements, 2017. Available at: https://www.iso.org/standard/66442.html. Accessed on: October 31, 2017.

Jozala AF, Geraldes DC, Tundisi, LL, Feitosa VA, Breyer CA, Cardoso SL, Mazzola PG, Oliveira-Nascimento L, Rangel-Yagui CO, Magalhães PO, Oliveira MA, Pessoa Jr A. Biopharmaceuticals from microorganisms: from production to purification. Braz J Microbiol, 2016; 47: 51-63.

Kuhnert P, Nicolet J, Frey J. Rapid and accurate Identification of Escherichia coli K-12 Strains. Appl Environ Microbiol, 1995; 61: 4135-4139.

Li D, Yu T, Zhang Y, Yang M, Li Z, Liu M, Qi R. Antibiotic resistance characteristics of environmental bacteria from an oxytetracycline production wastewater treatment plant and the receiving river. Appl Environ Microbiol, 2010; 76: 3444-3451.

Lundahl G. A Method of Increasing Test Range and Accuracy of Bioindicators: Geobacillus stearothermophilus Spores. PDA J Pharm Sci Technol, 2003; 57: 249-262.

Maheux AF, Dion-Dupont V, Bisson MA, Bouchard S, Rodriguez MJ. Detection of Escherichia coli colonies on confluent plates of chromogenic media used in membrane filtration. J Microbiol Methods, 2014; 97: 51-55.
Mannermaa J, Yliruusi J. Optimization of Moist Heat Sterilization. PDA J Pharm Sci Technol, 1993; 47: 306-310

Martinez JL. Environmental pollution by antibiotics and by antibiotic resistance determinants. Environ Pollut, 2009; 157: 2893-2902.

McLeod NP, Clifford M, Sutton JM. Evaluation of Novel Process Indicators for Rapid Monitoring of Hydrogen Peroxide Decontamination Processes. PDA J Pharm Sci Technol, 2017; 71: 393-404.

Miralles NF, Espín JD, Corchero JL, Vázquez E, Villaverde A. Microbial factories for recombinant pharmaceuticals. Microb Cell Fact, $2009 ; 1-8$

Rathore AS, Kapoor G. Application of process analytical technology for downstream purification of biotherapeutics. J Chem Technol Biotechnol, 2015; 90: 228-236.

Rosa PAJ, Ferreira IF, Azevedo AM, Aires-Barros MR. Aqueous two-phase systems: a viable platform in the manufacturing of biopharmaceuticals. J Chromatogr A, 2010; 1217: 2296-2305.

Simões GAR, Xavier MAS, Oliveira DA, Menezes EV, Magalhães SSG, Gandra JACD, Xavier AREO. Genetic markers for detection of Escherichia coli K-12 harboring ampicillin-resistance plasmid from an industrial wastewater treatment effluent pond. Genet Mol Res 2016; 15: 1-12.

Turner KM, Restaino L, Frampton EW. Efficacy of Chromocult Coliform Agar for Coliform and Escherichia coli Detection in Foods. J Food Prot, 2000; 63: 539-541.

USP $<61>$ Microbiological Examination of Nonsterile Products: Microbial Enumeration Tests. 2016. Available at: https:/hmc.usp.org/sites/ default/files/documents/HMC/GCs-Pdfs/c61.pdf. Accessed on: October 2, 2017.

Wang ZH, Yang JQ, Zhang DJ, Zhou J, Zhang CD, Su XR, Li TW. Composition and structure of microbial communities associated with different domestic sewage outfalls. Genet Mol Res, 2014; 13: 7542-7552.

How to cite this article:

Antunes GA, Gandra JACD, Moreira EA, Machado WCS, Magalhães SSG, Xavier MAS, Xavier AREO. Chromocult Coliform agar and duplex PCR assays as methodologies for tracking Escherichia coli $\mathrm{K} 12$ in industrial biotechnological processes. J App Pharm Sci, 2018; 8(03): 126-132. 